\title{
Quantum multimode model of elastic scattering from Bose Einstein condensates
}

\author{
P. Ziñ ${ }^{1}$, J. Chwedeńczuk ${ }^{1}$, A. Perez ${ }^{1}$, K. Rzążewski ${ }^{2}$ and M. Trippenbach ${ }^{1}$ \\ 1 Physics Department, Warsaw University, Hoża 69, \\ PL-00-681 Warsaw, Poland, ${ }^{2}$ Center for Theoretical Physics, \\ Polish Academy of Science, Al. Lotników 32/46, PL-02-668 Warsaw, Poland.
}

\begin{abstract}
Mean field approximation treats only coherent aspects of the evolution of a Bose Einstein condensate. However, in many experiments some atoms scatter out of the condensate. We study an analytic model of two counter-propagating atomic Gaussian wavepackets incorporating dynamics of incoherent scattering processes. Within the model we can treat processes of elastic collision of atoms into the initially empty modes, and observe how, with growing occupation, the bosonic enhancement is slowly kicking in. A condition for bosonic enhancement effect is found in terms of relevant parameters. Scattered atoms form a squeezed state that can be viewed as a multi-component condensate. Not only are we able to calculate the dynamics of mode occupation, but also the full statistics of scattered atoms.
\end{abstract}

A remarkably universal tool describing vast majority of experiments with the Bose Einstein condensates is the celebrated Gross-Pitaevskii equation [GPE]. It describes a coherent evolution of the atomic mean field. In the Hartree interpretation, its time-dependent version assumes that each atom of the system undergoes identical evolution. This is a good assumption since in typical experiments the wave-packet of the system contains many thousands of particles in the same state. To use a term borrowed from quantum optics, the time-dependent GPE describes stimulated processes. In some experiments 1], however, there is a clear evidence of spontaneous processes. For example, in a collision between two condensates, some atoms from colliding quantum matter droplets would inevitably scatter away from them. This is a loss process, which is not accounted for by the conventional GPE. Description of such phenomena calls for use of quantum fields instead of c-number wave-functions. This is not easy since, in general, field equations are nonlinear. Instead of quantum fields, several groups used classical stochastic fields to imitate quantum initiation of spontaneous processes 22. At this point it is hard to access the accuracy of these methods. Solid results so far has only been obtained within perturbation theory $[3,4,[5]$. It is the purpose of this Letter to present the first exact nonperturbative calculation of collisional losses, valid in the regime of Bose enhancement. Our model assumes spherical nonspreading Gaussians for the colliding wave-packets. No doubt it will serve as a benchmark test of validity of various approximate schemes including classical stochastic fields.

A system of Bosons interacting via contact potential is described by the Hamiltonian

$$
\begin{aligned}
\hat{\mathrm{H}} & =-\int \mathrm{d}^{3} r \hat{\Psi}^{\dagger}(\mathbf{r}, t) \frac{\hbar^{2} \nabla^{2}}{2 m} \hat{\Psi}(\mathbf{r}, t) \\
& +\frac{g}{2} \int \mathrm{d}^{3} r \hat{\Psi}^{\dagger}(\mathbf{r}, t) \hat{\Psi}^{\dagger}(\mathbf{r}, t) \hat{\Psi}(\mathbf{r}, t) \hat{\Psi}(\mathbf{r}, t),
\end{aligned}
$$

where $\hat{\Psi}(\mathbf{r})$ is a field operator satisfying equal time bosonic commutation relations, $m$ is the atomic mass and $g$ determines the strength of the inter-atomic interactions. Since the Hamiltonian (1I) is of the fourth order in $\hat{\Psi}$, the Heisenberg equation governing the evolution of the field will be nonlinear and thus, in general, analytically and numerically untractable. However, for some physical systems, a Bogoliubov approximation can be applied leading to linear Heisenberg equations. The idea underlying this approximation states that for some cases the field operator might be split into two parts $\psi$ and $\hat{\delta}$. First contribution describes macroscopically occupied field and since its fluctuations are usually small, its operator character might be dropped ( $\psi$ becomes a c-number wave-function satisfying GPE). The second part $\hat{\delta}$, representing fluctuations, will require full quantum mechanical treatment, but as long as we neglect its back-reaction on $\psi$ the evolution of $\hat{\delta}$ will be linear.

In this Letter we consider a process of collision of two strongly occupied Bose Einstein condensates. Initial state of the system consists of two counter-propagating atomic wave-packets and the "sea" of unoccupied modes. For such a system the Bogoliubov approximation can be applied. The splitting of the bosonic field is performed in the following manner:

$$
\hat{\Psi}(\mathbf{r}, t)=\psi_{Q}(\mathbf{r}, t)+\psi_{-Q}(\mathbf{r}, t)+\hat{\delta}(\mathbf{r}, t),
$$

where the subscript $\pm Q$ denotes the mean momentum of the colliding condensates. Upon inserting Eq. (2) into the Hamiltonian (11) one obtains a collection of different terms. We keep only those, that lead to creation or annihilation of a pair of particles

$$
\begin{aligned}
\mathrm{H} & =-\int \mathrm{d}^{3} r \hat{\delta}^{\dagger}(\mathbf{r}, t) \frac{\hbar^{2} \nabla^{2}}{2 m} \hat{\delta}(\mathbf{r}, t) \\
& +g \int \mathrm{d}^{3} r \hat{\delta}^{\dagger}(\mathbf{r}, t) \hat{\delta}^{\dagger}(\mathbf{r}, t) \psi_{Q}(\mathbf{r}, t) \psi_{-Q}(\mathbf{r}, t)+\text { H.c. }
\end{aligned}
$$

One can argue that such an approximation gives correct results if and only if the kinetic energy associated with the center-of-mass motion is much larger than the interaction energy per particle, $\hbar^{2} Q^{2} /(2 m) \gg g n$, where $\mathrm{n}$ is 
the average density of the particles in the condensates. Numerical proof of the above statement in the simplest case of two plane matter waves was given in 3]. This condition is readily fulfilled in current experiments [1, 6, 7] and all the results below are obtained in this regime.

In order to further simplify the dynamics we compare three characteristic timescales that appear in the problem; the collisional time, $t_{C}=(m \sigma) /(\hbar Q)$, the time it takes for each wave-packet to pass through its colliding partner, the linear dispersion time, $t_{L D}=m \sigma^{2} / \hbar$ [8], characteristic time of the spread of the wave-packet due to kinetic energy term (neglecting the nonlinearity), and nonlinear dispersion time, $t_{N D}=\sqrt{\pi^{3 / 2} m \sigma^{5} / g N}$, time of ballistic expansion in Thomas Fermi approximation [9]. Here each of the wave-packets has the radius of $\sigma$ and contains $N / 2$ atoms. The dynamics of our system depends on the relations between timescales defined above. Hence we introduce dimensionless parameters: $t_{L D} / t_{C}=\beta$ and $\left(t_{L D} / t_{N D}\right)^{2}=\alpha$. When the number of elastically scattered atoms is small in comparison with the total number of atoms in both wave-packets and both linear and nonlinear dispersion timescales are much longer than the collisional time $\left(\left(t_{L D} / t_{C}\right)=\beta \gg 1\right.$ and $\left.\left(t_{N D} / t_{C}\right)^{2}=\beta^{2} / \alpha \gg 1\right)$, we can neglect the change of shape of the macroscopically occupied functions $\psi_{Q}(\mathbf{r}, t)$ during the collision. In our model we use spherically symmetric Gaussian wave-functions

$$
\begin{aligned}
& \psi_{ \pm Q}(\mathbf{r}, t)=\sqrt{\frac{N}{2 \pi^{3 / 2} \sigma^{3}}} \exp \left[ \pm i Q x_{1}-\frac{i \hbar t Q^{2}}{2 m}\right] \times \\
& \times \exp \left[-\frac{1}{2 \sigma^{2}}\left(\left(x_{1} \mp \frac{\hbar Q t}{m}\right)^{2}+x_{2}^{2}+x_{3}^{2}\right)\right],
\end{aligned}
$$

where $\mathbf{r}=\left(x_{1}, x_{2}, x_{3}\right)$. In the dimensionless units, $\left(t \equiv t / t_{C}\right.$ and $x_{i} \equiv x_{i} / \sigma$, for $\left.i=1,2,3\right)$, the Heisenberg evolution equation of the field operator $\hat{\delta} \equiv \hat{\delta} \exp (i \beta t / 2)$ can be obtained upon substituting (4) into (3)

$$
i \beta \partial_{t} \hat{\delta}(\mathbf{r}, t)=-\frac{1}{2}\left(\Delta+\beta^{2}\right) \hat{\delta}(\mathbf{r}, t)+\alpha e^{-r^{2}-t^{2}} \hat{\delta}^{\dagger}(\mathbf{r}, t) .
$$

The above equation has spherical symmetry! Hence, we decompose $\hat{\delta}$ into the basis of spherical harmonics

$$
\hat{\delta}(\mathbf{r}, t)=\sum_{n, l, m} R_{n, l}(r) Y_{l m}(\theta, \phi) \hat{a}_{n, l, m}(t),
$$

where $\hat{a}_{n, l, m}$ are annihilation operators for a particle in the mode described by $n, l, m$ quantum numbers. There is still a freedom of choice with regards to the set of orthogonal functions $R_{n, l}(r)$. As we shall see below a good candidate is a set of eigenfunctions of spherically symmetric harmonic oscillator,

$$
R_{n, l}(r)=\sqrt{\frac{2 n ! a_{0}^{-3}}{\Gamma\left(l+n+\frac{3}{2}\right)}}\left(\frac{r}{a_{0}}\right)^{l} e^{-\frac{r^{2}}{2 a_{0}^{2}}} L_{n}^{l+\frac{1}{2}}\left(\frac{r^{2}}{a_{0}^{2}}\right),
$$

where $L_{n}^{l+\frac{1}{2}}(x)$ is the associated Laguerre polynomial 10 ] and $a_{0}$, a harmonic oscillator length, is an auxiliary free parameter that can be chosen to minimize the computational effort. The evolution of $\hat{a}_{n, l, m}(t)$ is described by

$$
\begin{gathered}
i \partial_{t} \hat{a}_{n, l, m}=\frac{E_{n, l}-\beta^{2}}{2 \beta} \hat{a}_{n, l, m}+D_{n, l} \hat{a}_{n-1, l, m}+ \\
D_{n+1, l} \hat{a}_{n+1, l, m}+\frac{\alpha}{\beta} \mathrm{e}^{-t^{2}} \sum_{n^{\prime}} C_{n, n^{\prime}, l} \hat{a}_{n^{\prime}, l,-m}^{\dagger},
\end{gathered}
$$

where coefficients $D_{n, l}=\sqrt{n(n+l+1 / 2)} /\left(2 \beta a_{0}^{2}\right)$, $E_{n, l}=(2 n+l+3 / 2) / a_{0}^{2}$ and

$$
\begin{aligned}
& C_{n, n^{\prime}, l}=\int_{0}^{\infty} r^{2} \mathrm{~d} r R_{n, l}(r) \exp \left(-r^{2}\right) R_{n^{\prime} l}(r)= \\
& =\sqrt{\frac{\Gamma\left(n+l+\frac{3}{2}\right) \Gamma\left(n^{\prime}+l+\frac{3}{2}\right)}{\Gamma\left(l+\frac{3}{2}\right)^{2} \Gamma(n+1) \Gamma\left(n^{\prime}+1\right)}}\left(1+a_{0}^{2}\right)^{-l-\frac{3}{2}} \times \\
& \times\left[\frac{a_{0}^{2}}{1+a_{0}^{2}}\right]^{n+n^{\prime}} F\left(-n,-n^{\prime}, l+\frac{3}{2}, 1 / a_{0}^{4}\right)
\end{aligned}
$$

Here $F(a, b, c, x)$ is a hypergeometric function [10]. Notice that all coupling coefficients are calculated analytically and the $\hat{a}_{n, l, m}$ operators for different $l$ and $m$ are decoupled. Moreover, equations (8) do not depend on quantum number $m$. With all these simplifications the linear system of equations (8) can be solved numerically.

The solution of the set of dynamical equations 8 for $\hat{a}_{n, l, m}$ contains the full information about the considered quantum system. In particular, we can reconstruct the operator $\hat{\delta}(\mathbf{r}, t)$, using decomposition defined in Eq. (6). The most straightforward observable quantity, the number of elastically scattered atoms as a function of time can be expressed in terms of the trace of the density matrix

$$
\begin{aligned}
\mathcal{S}(t)= & \int d^{3} r\left\langle\hat{\delta}^{\dagger}(\mathbf{r}, t) \hat{\delta}(\mathbf{r}, t)\right\rangle= \\
& =\sum_{n=0}^{\infty} \sum_{l=0}^{\infty}(2 l+1)\left\langle\hat{a}_{n, l, m}^{\dagger}(t) \hat{a}_{n, l, m}(t)\right\rangle,
\end{aligned}
$$

where $(2 l+1)$ accounts for the degeneracy of Eq. (8) with regards to the quantum number $m$ [17]. In the limit where $\alpha / \beta$ is small (notice that in Eq. (8), this coefficient multiplies the source term), $S(t)$ can be evaluated in the first order perturbation approximation giving [3]

$$
\mathcal{S}(t)=\frac{\pi \alpha^{2}}{16} \operatorname{erf}(\sqrt{2} t)
$$

The same result is obtained using imaginary scattering length method [4]. Quality of this approximation is illustrated in Fig

The bonus of having solved the full set of operator equations is that calculating full density matrix of the 


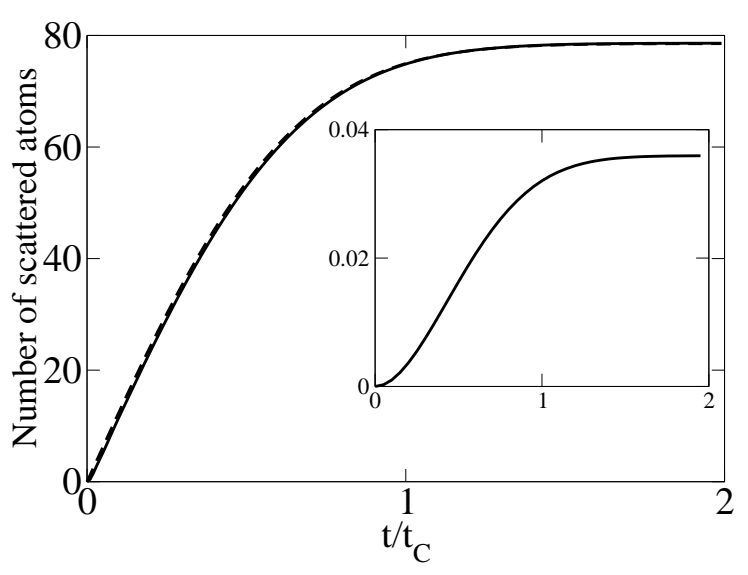

FIG. 1: Number of scattered atoms versus time in perturbative regime; dashed line - analytical result given by (11). Solid line - numerical result obtained from our model (using Eq. (10) for $\alpha=20$ and $\beta=60$. The inset shows the time evolution of the largest eigenvalue of the density matrix.

system of scattered atoms $\left(\rho\left(\mathbf{r}, \mathbf{r}^{\prime}, t\right)=\left\langle\hat{\delta}^{\dagger}(\mathbf{r}, t) \hat{\delta}\left(\mathbf{r}^{\prime}, t\right)\right\rangle\right)$ or even higher order correlation functions is just as easy as finding $\mathcal{S}(t)$. In the basis (6), due to the decoupling property, density matrix can be written as a direct product of $\rho_{n, n^{\prime}, l, m}=\left\langle\hat{a}_{n, l, m}^{\dagger}(t) \hat{a}_{n^{\prime}, l, m}(t)\right\rangle$ matrices, for different $l$ and $m$. In the inset of Fig प we present the time evolution of the largest of the eigenvalues of the density matrix $\rho\left(\mathbf{r}, \mathbf{r}^{\prime}, t\right)$. Due to the normalization of the density matrix, $\sum_{i} \lambda_{i}(t)=\mathcal{S}(t)$, where $\lambda_{i}$ are the eigenvalues of the density matrix, the inset of Fig 1 shows that for $\alpha=20, \beta=60$ there is much less than one particle even in the mostly populated eigenmode.

Figure 2 shows analogous comparison between perturbative solution (11) and formula (10) in the regime of parameters where the perturbation theory is expected to fail (the criterion for bosonic enhancement is $\alpha / \beta>1$ 12]). The figure shows that until some critical time, approximately equal to $0.2 t_{C}$, both the perturbative and full solutions agree very well. At this critical time the formula (10) exceeds the perturbative solution and the difference between curves rapidly grows in time. At the same time the biggest eigenvalue of the density matrix of the system reaches one, which means that there is one particle in the mostly populated eigenmode. This observation gives explanation to the growing discrepancy between two curves shown in Fig 2 Once approximately one atom is scattered into one of the eigenmodes of the density matrix the probability of scattering another atom into this mode grows rapidly. This is due to bosonic statistics of the scattered atoms and is called bosonic enhancement effect.

An interesting information about the system might be obtained upon analyzing the largest eigenvalues of

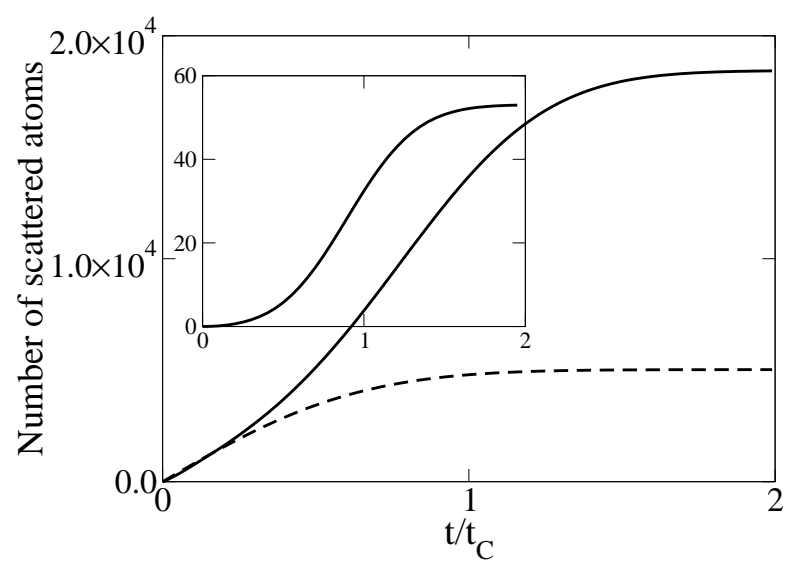

FIG. 2: Number of scattered atoms versus time in nonperturbative regime where the bosonic enhancement occurs. Dashed line - analytical result given by (11). Solid line - numerical result obtained from (10). Parameters are: $\alpha=160$, $\beta=40$. The inset shows the time evolution of the biggest eigenvalue of the density matrix.

$\rho_{n, n^{\prime}, l, m}$ for each quantum number $l$. Figure 3 juxtaposes these eigenvalues as a function of $l$, for the case with bosonic enhancement. The plot shows that the density matrix has several eigenvalues of the same order. Such a system is similar to quasi-condensate, in contrast to the commonly used definition of the condensate as described by a density matrix having one dominant eigenvalue [1].

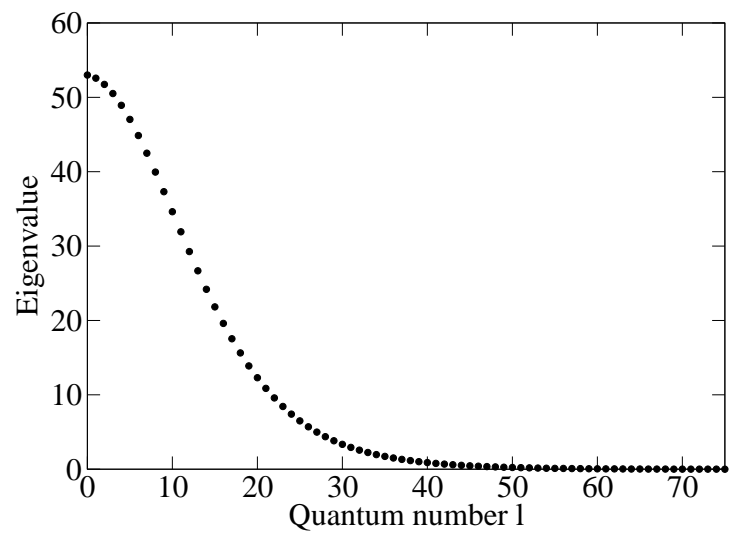

FIG. 3: The biggest eigenvalue of the density matrix for different $l$ for $\alpha=160$ and $\beta=40$, at time $t=2 t_{C}$. Several eigenvalues of the same order indicate the presence of the quasi-condensate.

From the experimental point of view, coherence properties of the scattered atoms are of great importance. These properties are best characterized by the correlation functions. In particular, the first and second order 


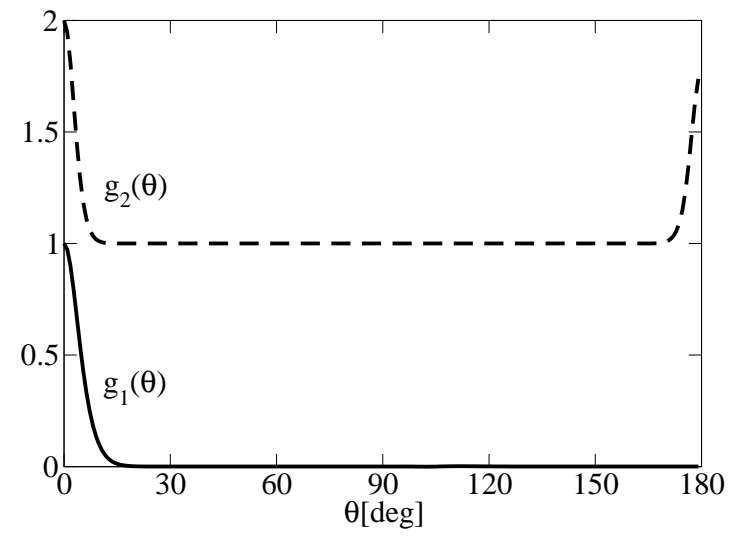

FIG. 4: First and second order correlation functions in momentum space $g_{1}\left(\mathbf{k}, \mathbf{k}^{\prime}\right)$ and $g_{2}\left(\mathbf{k}, \mathbf{k}^{\prime}\right)$ for $|\mathbf{k}|=\left|\mathbf{k}^{\prime}\right|=Q$, as a function of relative azimuthal angle $\theta$ at $t=2 t_{C}$ for $\alpha=160$ and $\beta=40$.

correlation functions can be measured in experiment. In one of the most commonly used method, time-of-flight measurement, the momentum distribution of the system is obtained. Thus here we calculate the first and second order correlation functions in momentum space using

$$
g_{1}\left(\mathbf{k}, \mathbf{k}^{\prime}, t\right)=\frac{\left\langle\hat{\delta}^{\dagger}(\mathbf{k}, t) \hat{\delta}\left(\mathbf{k}^{\prime}, t\right)\right\rangle}{\sqrt{\left\langle\hat{\delta}^{\dagger}(\mathbf{k}, t) \hat{\delta}(\mathbf{k}, t)\right\rangle\left\langle\hat{\delta}^{\dagger}\left(\mathbf{k}^{\prime}, t\right) \hat{\delta}\left(\mathbf{k}^{\prime}, t\right)\right\rangle}}(12)
$$

for the former, and

$$
g_{2}\left(\mathbf{k}, \mathbf{k}^{\prime}, t\right)=\frac{\left\langle\hat{\delta}^{\dagger}(\mathbf{k}, t) \hat{\delta}^{\dagger}\left(\mathbf{k}^{\prime}, t\right) \hat{\delta}\left(\mathbf{k}^{\prime}, t\right) \hat{\delta}(\mathbf{k}, t)\right\rangle}{\left\langle\hat{\delta}^{\dagger}(\mathbf{k}, t) \hat{\delta}(\mathbf{k}, t)\right\rangle\left\langle\hat{\delta}^{\dagger}\left(\mathbf{k}^{\prime}, t\right) \hat{\delta}\left(\mathbf{k}^{\prime}, t\right)\right\rangle}
$$

for the latter. Due to spherical symmetry of Heisenberg equation for $\hat{\delta}$, the momentum density $\left\langle\hat{\delta}^{\dagger}(\mathbf{k}, t) \hat{\delta}(\mathbf{k}, t)\right\rangle$ is spherically symmetric as well. Moreover, since the Hamiltonian (3) is quadratic in $\hat{\delta}$ and the initial state is a vacuum state, than, in Schrödinger picture, at any later time $t$ the state of scattered atoms is a multimode squeezed state 18. According to general properties of multimode squeezed states, the $n$-th order correlation function $g_{n}\left(\mathbf{k}, \mathbf{k}^{\prime}\right)$ for $\mathbf{k}=\mathbf{k}^{\prime}$ is equal to $n$ !. It is confirmed by our numerical results. The solid line in Fig 4 shows the first order correlation function (12) plotted for fixed length of the $\mathbf{k}$ and $\mathbf{k}^{\prime}$ vectors $\left(|\mathbf{k}|=\left|\mathbf{k}^{\prime}\right|=Q\right)$ as a function of relative angle $\theta$. As expected, for $\theta=0$ the condition, $g_{1}(\mathbf{k}, \mathbf{k})=1$ is satisfied. Also, the limited coherence angle, due to spontaneous initiation of scattering process is clearly visible. The dashed line Fig 4 shows the second order correlation function (13). Once again, a prediction $g_{2}(\mathbf{k}, \mathbf{k})=2$ is met. As Fig 4 shows, the $g_{2}$ function reveals strong correlation between atoms scattered in direction $\mathbf{k}$ and $\mathbf{- k}$ which corresponds to relative angle $\theta=180^{\circ}$. This is an intuitive result, since atoms get scattered in pairs in such a way that the momentum and energy conservation laws are satisfied. Finally, the width of the correlation peak of $g_{2}$ in the forward direction in the perturbative regime scales as $1 / \beta$, which is proportional to the size of colliding wave-packets [12]. This is in analogy to Hanburry-Brown and Twiss method of estimating sizes of distant stars by measuring intensityintensity correlation function [13 and relating densitydensity correlation of $\pi$-mesons to the size of fireball in high energy collision of hadrons [14].

In conclusion, upon analyzing the quantum model of two counter-propagating atomic Gaussian wave-packets we get a deeper insight into processes of elastic collision losses of atoms and are able to study the transition from spontaneous regime to the bosonic enhancement. Scattered atoms form a squeezed state that can be viewed as a multi-component condensate. Within this model in principle all order correlation functions are accessible and hence it has a high predictive power.

The authors acknowledge support from KBN Grant 2P03 B4325 (J. Ch., P.Z.), Polish Ministry of Scientific Research and Information Technology under grant PBZMIN-008/P03/2003 (M. T., K.R.).

[1] M. Kozuma et al., Phys. Rev. Lett. 82, 871 (1999); J. M. Vogels, K. Xu, and W. Ketterle, Phys. Rev. Lett. 89, 020401 (2002)

[2] J. Chwedeńczuk, M. Trippenbach and K. Rzążewski, J. Phys. B, L391, 37 (2004); A.A. Norrie, R.J. Ballagh and C.W. Gardiner, cond-mat/0403378

[3] R. Bach, M. Trippenbach and K. Rzążewski, Phys. Rev. A 65063605 (2002)

[4] Y. B. Band et al., Phys. Rev. Lett. 84, 5462 (2000)

[5] V. A. Yurovsky, Phys. Rev. A 65033605 (2002)

[6] J. Stenger et al., Phys. Rev. Lett. 82, 4569 (1999).

[7] A. P.Chikkatur et al., Phys. Rev. Lett. 85, 483 (2000).

[8] M. Trippenbach, Y. B. Band, and P. S. Julienne, Phys. Rev. A 62, 023608 (2000)

[9] Y. Castin and R. Dum, Phys. Rev. Lett. 77, 5315-5319 (1996); Yu. Kagan, E. L. Surkov, and G. V. Shlyapnikov, Phys. Rev. A 55, R18-R21 (1997)

[10] M. Abramovich, I. A. Stegun, "Handbook of Mathematical Functions With Formulas, Graphs and Mathematical Tables", Dover Publications 1974.

[11] V.A. Zagrebnov, J.-B. Bru, Physics Reports 350, 291$434(2001)$

[12] J. Chwedeczuk et al., in preparation.

[13] R. Hanburry-Brown and R. Q. Twiss, Phil. Mag. 45, 633 (1954)

[14] G. I. Kopylov, Phys. Lett. 50B, 472 (1974)

[15] T. Kohler and K. Burnett, Phys. Rev A 65, 033601 (2002)

[16] L. Deng et al, Nature (London) 398, 218 (1999)

[17] The expectation value in equation (10) and below it are taken in the initial vacuum state $|0\rangle$, satisfying the condition $\hat{a}_{n, l, m}(0)|0\rangle=0$ for all $n, l$ and $m$.

[18] X. Ma and W. Rhodes, Phys. Rev A 41, 4625 (1990) 